REPRESENTATION THEORY

An Electronic Journal of the American Mathematical Society

Volume 3, Pages 435-443 (November 9, 1999)

S $1088-4165(99) 00086-2$

\title{
IRREDUCIBLE REPRESENTATIONS OF SOLVABLE LIE SUPERALGEBRAS
}

\author{
ALEXANDER SERGEEV
}

\begin{abstract}
The description of irreducible finite dimensional representations of finite dimensional solvable Lie superalgebras over complex numbers given by $\mathrm{V}$. Kac is refined. In reality these representations are not just induced from a polarization but are twisted ones, as infinite dimensional representations of solvable Lie algebras. Various cases of irreducibility (general and of type Q) are classified.
\end{abstract}

\section{INTRODUCTION}

Hereafter the ground field is $\mathbb{C}$ and all the modules and superalgebras are finite dimensional; $\mathbb{Z} / 2=\{\overline{0}, \overline{1}\}$ and $\mathfrak{g}=\mathfrak{g}_{\overline{0}} \oplus \mathfrak{g}_{\overline{1}}$ is a solvable Lie superalgebra.

The description of irreducible representations of solvable Lie superalgebras given in $[\mathrm{K}]$ (Theorem 7) contains an error. In reality, to give such a description one has to imitate the description of infinite dimensional solvable Lie algebras [D], i.e., we must consider twisted induced representations. In what follows I give a correct description of irreducible representations of solvable Lie superalgebras. I also show where a mistake crept into $[\mathrm{K}]$ and give a counterexample to Theorem 7 from $[\mathrm{K}]$.

The proof given in what follows was delivered at Leites' Seminar on Supermanifolds in 1983 and is preprinted in [L] in a form considerably edited by I. Shchepochkina and D. Leites. My acknowledgements are due to them and also to the Department of Mathematics of Stockholm University that financed publication of [L].

\section{Main Result}

1.1. Polarizations. Set $L=\left\{\lambda \in \mathfrak{g}^{*}: \lambda\left(\mathfrak{g}_{\overline{1}}\right)=0\right.$ and $\left.\lambda\left(\left[\mathfrak{g}_{\overline{0}}, \mathfrak{g}_{\overline{0}}\right]\right)=0\right\}$. Recall that a superspace is a $\mathbb{Z} / 2$-graded space $V=V_{\overline{0}} \oplus V_{\overline{1}}$ and its superdimension is the pair $\left(\operatorname{dim} V_{\overline{0}}, \operatorname{dim} V_{\overline{1}}\right)$. By the usual abuse of language $\lambda \in L$ denotes a character and also the $(1,0)$-dimensional representation of the Lie algebra $\mathfrak{g}_{\overline{0}}$ determined by the character $\lambda$. Every functional $\lambda \in L$ determines a symmetric form $f_{\lambda}$ on $\mathfrak{g}_{\overline{1}}$ by the formula $f_{\lambda}\left(\xi_{1}, \xi_{2}\right)=\lambda\left(\left[\xi_{1}, \xi_{2}\right]\right)$.

A subalgebra $\mathfrak{h} \subset \mathfrak{g}$ is called a polarization for $\lambda \in L$ if $\lambda([\mathfrak{h}, \mathfrak{h}])=0, \mathfrak{h} \supset \mathfrak{g}_{\overline{0}}$ and $\mathfrak{h}_{\overline{1}}$ is a maximal fully isotropic subspace for $f_{\lambda}$.

Lemma. For every $\lambda \in L$ there exists a polarization $\mathfrak{h}$.

Proof follows from Lemma 2.4.

Received by the editors November 4, 1998 and, in revised form, September 8, 1999.

1991 Mathematics Subject Classification. Primary 17A70; Secondary 17 B30.

Key words and phrases. Solvable Lie superalgebras.

(c)1999 American Mathematical Society 
1.2. Twisted representations. If $\mathfrak{h}$ is a polarization for $\lambda \in L$, then, clearly, $\lambda$ determines a (1,0)-dimensional representation of $\mathfrak{h}$. Let $\mathfrak{h} \subset \mathfrak{g}$ be a Lie subsuperalgebra that contains $\mathfrak{g}_{\overline{0}}$. Define a functional $\theta_{\mathfrak{h}} \in L$ by setting

$$
\theta_{\mathfrak{h}}(g)=\left\{\begin{array}{cc}
-\frac{1}{2} \operatorname{tr}_{\mathfrak{g} / \mathfrak{h}}(\operatorname{ad} g) & \text { for } g \in \mathfrak{g}_{\overline{0}} \\
0 & \text { for } g \in \mathfrak{g}_{\overline{1}} .
\end{array}\right.
$$

Note that $\theta_{\mathfrak{h}}([\mathfrak{h}, \mathfrak{h}])=0$. Therefore, $\theta_{\mathfrak{h}}$ is a character of a $(1,0)$-dimensional representation of $\mathfrak{h}$.

Let $\mathfrak{h}$ be a polarization for $\lambda \in L$. Define the twisted (by the character $\theta_{\mathfrak{h}}$ ) induced and coinduced representations by setting

$$
\begin{gathered}
I_{\mathfrak{h}}^{\mathfrak{g}}(\lambda)=\operatorname{Ind}_{\mathfrak{h}}^{\mathfrak{g}}\left(\lambda+\theta_{\mathfrak{h}}\right)=U(\mathfrak{g}) \otimes_{U(\mathfrak{h})}\left(\lambda+\theta_{\mathfrak{h}}\right) ; \\
C I_{\mathfrak{h}}^{\mathfrak{g}}(\lambda)=\operatorname{Coind}_{\mathfrak{h}}^{\mathfrak{g}}\left(\lambda-\theta_{\mathfrak{h}}\right)=\operatorname{Hom}_{U(\mathfrak{h})}\left(U(\mathfrak{g}), \lambda-\theta_{\mathfrak{h}}\right) .
\end{gathered}
$$

Lemma. 1) $I_{\mathfrak{h}}^{\mathfrak{g}}(\lambda)$ is finite dimensional and irreducible.

2) $I_{\mathfrak{h}}^{\mathfrak{g}}(\lambda)$ does not depend on the choice of a polarization $\mathfrak{h}$; therefore, notation $I(\lambda)\left(=I_{\mathfrak{h}}^{\mathfrak{g}}(\lambda)\right.$ for some $\left.\mathfrak{h}\right)$ is well-defined.

3) $C I(\lambda) \cong I(\lambda)$.

For the proof see Corollaries 3.3 and 4.3.

1.3. Main Theorem. Let $Z=\{(\lambda, \mathfrak{h}): \lambda \in L$ and $\mathfrak{h}$ is a polarization for $\lambda\}$. Define an equivalence relation on $Z$ by setting

$$
(\lambda, \mathfrak{h}) \sim(\mu, \mathfrak{t}) \Longleftrightarrow \lambda-\theta_{\mathfrak{h}}=\mu-\theta_{\mathfrak{t}} .
$$

Clearly, this relation is well-defined.

Recall ([BL]) that the representation of a Lie superalgebra $\mathfrak{g}$ is called irreducible of $G$-type if it has no invariant subspaces; it is called irreducible of $Q$-type if it has no invariant subsuperspaces. Recall also that to every superspace $V=V_{\overline{0}} \oplus V_{\overline{1}}$ the change of parity functor $\Pi$ assigns the superspace $\Pi(V)$ such that $\Pi(V)_{\bar{i}}=V_{\bar{i}+\overline{1}}$. Observe that the modules $I(\lambda)$ and $\Pi(I(\lambda))$ are not isomorphic as $\mathfrak{g}$-modules (unless they are of $Q$-type); they are always isomorphic, however, as $\mathfrak{g}_{0}$-modules.

Theorem. 1) Every irreducible finite dimensional representation of $\mathfrak{g}$ is isomorphic up to application of the change of parity functor $\Pi$ to a representation of the form $I(\lambda)$ for some $\lambda$.

2) The map $\lambda \mapsto I(\lambda)$ is (up to П) a one-to-one correspondence between elements of $L$ and the irreducible finite dimensional representations of $\mathfrak{g}$.

3) Let $(\lambda, \mathfrak{h}),(\mu, \mathfrak{t}) \in Z$. Then $\operatorname{Ind}_{\mathfrak{h}}^{\mathfrak{g}}(\lambda) \cong \operatorname{Ind}_{\mathfrak{t}}^{\mathfrak{g}}(\mu)$ if and only if $(\lambda, \mathfrak{h}) \sim(\mu, \mathfrak{t})$.

4) If $\mathrm{rk} f_{\lambda}$ is even, then $I(\lambda)$ is a $G$-type representation; if $\operatorname{rk} f_{\lambda}$ is odd, then $I(\lambda)$ is a $Q$-type representation.

For the proof see sections $3.3,3.5,4.2$ and 4.3 .

Remark. For examples of irreducible representations of dimension $>1$ of solvable Lie superalgebras (and interesting examples of the latter) see [Shch].

\section{Prerequisites for the proof of Main Theorem}

Let $\mathfrak{k} \subset \mathfrak{g}$ be a subsuperalgebra, $\operatorname{codim} \mathfrak{k}=(0,1)$, and $\mu$ the character of the representation of $\mathfrak{g}_{\overline{0}}$ in $\mathfrak{g} / \mathfrak{k}$.

2.1. Lemma. $\mu$ is a character of $\mathfrak{g}$. 
Proof. Let $\xi \in \mathfrak{g}$ and $\xi \notin \mathfrak{k}$. Since in $\mathfrak{g} / \mathfrak{k}$ there is a $\mathfrak{k}$-action, it suffices to prove that $\mu([\mathfrak{k}, \xi])=\mu([\xi, \xi])=0$. By the Jacobi identity $[[\xi, \xi], \xi]=0$ which proves that $\mu([\xi, \xi])=0$. Let $\eta \in \mathfrak{k}_{\overline{1}}$. Then $[[\eta, \xi], \xi]=\frac{1}{2}[\eta,[\xi, \xi]] \in \mathfrak{k}$ and, therefore, $\mu\left(\left[\mathfrak{k}_{\overline{1}}, \xi\right]\right)=0$.

2.2. Corollary. Let $\mathfrak{k} \subset \mathfrak{k}_{1}$ be subalgebras in $\mathfrak{g}$ such that both contain $\mathfrak{g}_{\overline{0}}$ and such that $\operatorname{dim} \mathfrak{k}_{1} / \mathfrak{k}=(0,1)$. Let $\lambda$ be the character of an irreducible factor of $\mathfrak{g} / \mathfrak{k}$ considered as a $\mathfrak{g}_{\overline{0}}$-module. Then $\lambda$ is a character of $\mathfrak{k}_{1}$.

Proof. Let $\operatorname{dim} \mathfrak{g} / \mathfrak{k}=(0, l)$; we will induct on $l$. If $l=1$, the statement of Corollary holds thanks to Lemma 2.1 .

Let $l>1$ and $\mathfrak{k}_{2}$ be a subalgebra of $\mathfrak{g}$ such that $\operatorname{dim} \mathfrak{g} / \mathfrak{k}_{2}=(0,1)$ and $\mathfrak{k}_{2} \supset \mathfrak{k}_{1}$. Any irreducible factor of $\mathfrak{g} / \mathfrak{k}$ is a factor of either $\mathfrak{k}_{2} / \mathfrak{k}$ or $\mathfrak{g} / \mathfrak{k}_{2}$. In the first case Corollary holds by the inductive hypothesis. In the second case let $\lambda$ be the character of an irreducible factor of $\mathfrak{g} / \mathfrak{k}_{2}$. Then $\lambda$ is a character of $\mathfrak{k}_{2}$; hence, a character of $\mathfrak{k}_{1}$.

2.3. Corollary ([K], Prop. 1.3.3, p. 25). A Lie superalgebra $\mathfrak{g}=\mathfrak{g}_{\overline{0}} \oplus \mathfrak{g}_{\overline{1}}$ is solvable if and only if is $\mathfrak{g}_{\overline{0}}$ is also.

Proof. Here is an independent proof. We induct on $l=\operatorname{dim} \mathfrak{g}_{\overline{1}}$. If $l=0$, the statement is obvious. Let $l>0$. Set

$$
\tilde{\mathfrak{g}}=\left[\mathfrak{g}_{\overline{0}}, \mathfrak{g}_{\overline{0}}\right] \oplus\left[\mathfrak{g}_{\overline{1}}, \mathfrak{g}_{\overline{1}}\right] \oplus \mathfrak{g}_{\overline{1}} .
$$

Since $[\mathfrak{g}, \mathfrak{g}] \subset \tilde{\mathfrak{g}}$, it suffices to demonstrate that $\tilde{\mathfrak{g}}$ is solvable. Let $\mathfrak{h} \subset \mathfrak{g}$ and $\operatorname{dim} \mathfrak{g} / \mathfrak{h}=(0,1)$. By Lemma 2.1 we see that $\left[\tilde{\mathfrak{g}}_{\overline{0}}, \mathfrak{g}_{\overline{1}}\right] \subset \mathfrak{h}_{\overline{1}}$. Hence, $[\tilde{\mathfrak{g}}, \tilde{\mathfrak{g}}] \subset \tilde{\mathfrak{g}}_{\overline{0}} \oplus \mathfrak{h}_{\overline{1}}$. By the inductive hypothesis, $\tilde{\mathfrak{g}}_{\overline{0}} \oplus \mathfrak{h}_{\overline{1}}$ is a solvable Lie superalgebra, hence, so is $\mathfrak{g}$.

The converse statement is obvious.

2.4. Lemma. Let $W$ be a finite dimensional $\mathfrak{g}$-module, $f$ a symmetric $\mathfrak{g}$-invariant form on $W$ and $V$ a $\mathfrak{g}$-invariant fully isotropic subspace. Then there exists a maximal $\mathfrak{g}$-invariant $f$-isotropic subspace in $W$ containing $V$.

Proof. Without loss of generality we may assume that $f$ is nondegenerate. Suppose first that $\operatorname{rk} f=\operatorname{dim} W=2 l$ is even.

i) Let us prove first that $W$ contains a nonzero isotropic $\mathfrak{g}$-invariant one-dimensional subspace. Since $\mathfrak{g}$ is solvable, there exists a $w \in W$ such that $x w=\lambda(x) w$ for any $x \in \mathfrak{g}$. If $f(w, w)=0$, we are done. If $f(w, w) \neq 0$, then the invariance implies that

$$
0=f(x w, w)+f(w, x w)=2 \lambda(x) f(w, w) .
$$

Therefore, $\lambda(x)=0$ and $w$ is a $\mathfrak{g}$-invariant. Furthermore, $W=\operatorname{Span}(w) \oplus W_{1}$, where $W_{1}=\operatorname{Span}(w)^{\perp}$.

In $W_{1}$, select a one-dimensional $\mathfrak{g}$-invariant subspace $\operatorname{Span}\left(w_{1}\right)$. If $f\left(w_{1}, w_{1}\right)=$ 0 , we are done. If $f\left(w_{1}, w_{1}\right) \neq 0$, the above arguments show that $w_{1}$ is a $\mathfrak{g}$ invariant. Then $w_{2}=w+\alpha w_{1}$ is an isotropic and $\mathfrak{g}$-invariant vector for $\alpha=$ $\sqrt{-f(w, w) / f\left(w_{1}, w_{1}\right)}$.

ii) Now let us induct on $l$. If $l=1$, let us apply step i). If $l>1$ we may assume, thanks to i), that $V \neq 0$. If $V=V^{\perp}$, we are done. But if $V \neq V^{\perp}$, then $V \subset V^{\perp}$, since $V$ is fully isotropic; moreover, the restriction of $f$ onto $V^{\perp} / V$ is nondegenerate. The equality $\operatorname{dim} V+\operatorname{dim} V^{\perp}=2 l \operatorname{implies}$ that $\operatorname{dim} V^{\perp} / V$ is even. Therefore, by induction we prove that $V^{\perp} / V$ contains a maximal $\mathfrak{g}$-invariant fully 
isotropic subspace $\bar{U}$. But then its preimage $U$ in $V^{\perp}$ is a maximal fully isotropic $\mathfrak{g}$-invariant subspace of $W$ containing $V$.

The case $\operatorname{rk} f=\operatorname{dim} W=2 l+1$ is treated similarly.

2.5. Corollary. If $\mathfrak{h}$ is a polarization for $\lambda \in L, \mathfrak{n}$ the kernel of $f_{\lambda}$ and $\lambda_{1}, \ldots$, $\lambda_{l}$ are characters of irreducible subfactors of $\mathfrak{g}_{\overline{1}} / \mathfrak{n}$ considered as a $\mathfrak{g}_{\overline{0}}$-module, then $\mathfrak{h}$ is also polarization for $\mu=\lambda+\alpha_{1} \lambda_{1}+\cdots+\alpha_{l} \lambda_{l}$ for any $\alpha_{1}, \ldots, \alpha_{l} \in \mathbb{C}^{*}$.

Proof. Since $f_{\lambda}$ determines a nondegenerate $\mathfrak{g}_{0}$-invariant pairing $\mathfrak{h}^{\perp} / \mathfrak{n} \times \mathfrak{g}_{\overline{1}} / \mathfrak{h} \longrightarrow \mathbb{C}$, the characters $\lambda_{1}, \ldots, \lambda_{l}$ coincide, up to a sign, with characters of irreducible factors of $\mathfrak{g}_{\overline{1}} / \mathfrak{h}$. But the latter space is a $\mathfrak{h}$-module, so $\lambda_{i}\left(\left[\mathfrak{h}_{\overline{1}}, \mathfrak{h}_{\overline{1}}\right]\right)=0$ and, therefore, $\mathfrak{h}_{\overline{1}}$ is fully isotropic for $f_{\mu}$.

If $\mathfrak{h}_{\overline{1}}$ is a maximal fully isotropic subspace for $f_{\mu}$, we are done. Otherwise, i.e., if $\mathfrak{h}_{\overline{1}}$ is not a maximal fully isotropic subspace for $f_{\mu}$, select a $\mathfrak{g}_{\overline{0}}$-invariant subspace $\mathfrak{b}_{\overline{1}}$ of $\mathfrak{g}_{\overline{1}}$ distinct from $\mathfrak{h}_{\overline{1}}$ containing $\mathfrak{h}_{\overline{1}}$ and isotropic with respect to $f_{\mu}$.

Next, in the module $\mathfrak{b}_{\overline{1}} / \mathfrak{h}_{\overline{1}}$ select a one-dimensional $\mathfrak{g}_{\overline{0}}$-invariant subspace $\operatorname{Span}(\bar{\xi})$, where $\xi \in \mathfrak{b}_{\overline{1}}$. Then $\mathfrak{k}_{\overline{1}}=\mathfrak{h}_{\overline{1}} \oplus \operatorname{Span}(\xi)$ is fully isotropic with respect to $f_{\mu}$ and $\mathfrak{k}=\mathfrak{g}_{\overline{0}} \oplus \mathfrak{h}_{\overline{1}}$ is a subalgebra of $\mathfrak{g}$. Then $\lambda_{i}\left(\left[\mathfrak{k}_{\overline{1}}, \mathfrak{k}_{\overline{1}}\right]\right)=0$ by Corollary 2.2. But $\lambda=\mu-\alpha_{1} \lambda_{1}-\cdots-\alpha_{l} \lambda_{l}$; hence, $\lambda\left(\left[\mathfrak{k}_{\overline{1}}, \mathfrak{k}_{\overline{1}}\right]\right)=0$. In other words, $\mathfrak{k}_{\overline{1}}$ is fully isotropic with respect to $f_{\lambda}$. But this contradicts the maximality of $\mathfrak{h}_{\overline{1}}$.

The following two statements are standard, so their proofs are omitted.

2.6. Lemma. Let $\mathfrak{k} \subset \mathfrak{g}$ be a Lie subsuperalgebra, $\operatorname{dim} \mathfrak{g} / \mathfrak{k}=(0,1)$. If $(V, \rho)$ is an irreducible representation of $\mathfrak{k}$ in a superspace $V$, then $W=\operatorname{Ind}_{\mathfrak{k}}^{\mathfrak{g}}(V)$ is reducible if and only if $V$ admits $a \mathfrak{g}$-module structure that extends $\rho$.

2.7. Lemma (see $[\mathrm{K}]$, Lemma 5.2 .2 b). Let $\mathfrak{k} \subset \mathfrak{g}$ be a Lie subsuperalgebra, $\operatorname{dim} \mathfrak{g} / \mathfrak{k}$ $=(0,1)$. If $W$ is an irreducible $\mathfrak{g}$-module and $V \subset W$ is an irreducible proper $\mathfrak{k}$ submodule, then $W=\operatorname{Ind}_{\mathfrak{k}}^{\mathfrak{g}}(V)$.

\section{Description of IRREDuCIBle Modules}

3.1. Proposition. Let $\lambda \in L$, let $\mathfrak{p}=\mathfrak{g}_{0} \oplus \mathfrak{p}_{\overline{1}}$ be a polarization for $\lambda$, let $\mathfrak{n}$ be the kernel of $f_{\lambda}$ and $F \subset \mathfrak{p}_{\overline{1}}$ a subspace such that $\mathfrak{p}_{\overline{1}}=F \oplus \mathfrak{n}$. Define $\xi_{0}$ as follows. If $\mathrm{rk} f_{\lambda}$ is even, then we set $\xi_{0}=0$. If $\mathrm{rk} f_{\lambda}$ is odd, choose $\xi_{0}$ from $\mathfrak{p}_{\overline{1}}^{\perp}$ so that $\xi_{0} \notin \mathfrak{p}_{\overline{1}}$. Let $x v=\lambda(x) v$ be a one-dimensional representation of $\mathfrak{p}$ in $V=\operatorname{Span}(v)$. Denote $I_{\mathfrak{p}}^{\mathfrak{g}}(\lambda)=\operatorname{Ind}_{\mathfrak{p}}^{\mathfrak{g}}(V)$.

If $u \in I_{\mathfrak{p}}^{\mathfrak{g}}(\lambda)$ and $F u=0$, then $u \in \operatorname{Span}\left(v, \xi_{0} v\right)$.

Proof. Induction on rk $f_{\lambda}$. If $\mathrm{rk} f_{\lambda}=0$, then $F=0$ and the statement is obvious.

Let $\operatorname{rk} f_{\lambda}>0$. Select a subalgebra $\mathfrak{h} \subset \mathfrak{p}$ such that $\operatorname{dim} \mathfrak{g}_{\overline{1}} / \mathfrak{h}_{\overline{1}}=1$. Then two cases are possible: $\mathfrak{h}_{\overline{1}}^{\perp} \not \subset \mathfrak{h}_{\overline{1}}$ and $\mathfrak{h}_{\overline{1}}^{\perp} \subset \mathfrak{h}_{\overline{1}}$.

i) $\mathfrak{h}_{\overline{1}}^{\perp} \not \subset \mathfrak{h}_{\overline{1}}$. Then $\mathfrak{g}_{\overline{1}}=\mathfrak{h}_{\overline{1}} \oplus \operatorname{Span}(\xi)$, where $\xi \perp \mathfrak{h}_{\overline{1}}$. Hence, $\xi \perp \mathfrak{p}_{\overline{1}}$ and $\xi \notin \mathfrak{p}_{\overline{1}}$. Therefore, we may assume that $\xi=\xi_{0}$ and rk $f_{\lambda}$ is an odd number. Clearly, $\mathfrak{p}$ is a polarization for the restriction $f_{\lambda}$ onto $\mathfrak{h}_{\overline{1}}$ and $\mathrm{rk} f_{\lambda}$ is an even number. Furthermore,

$$
\operatorname{Ind}_{\mathfrak{p}}^{\mathfrak{g}}(\lambda)=\operatorname{Ind}_{\mathfrak{p}}^{\mathfrak{h}}(\lambda) \oplus \xi_{0} \operatorname{Ind}_{\mathfrak{p}}^{\mathfrak{g}}(\lambda) .
$$

Let $u=u_{0}+\xi_{0} u_{1} \in \operatorname{Ind}_{\mathfrak{p}}^{\mathfrak{g}}(\lambda)$ and $p u=0$ for any $p \in F$. Then

$$
0=p u=p u_{0}+\left[p, \xi_{0}\right] u_{1}+\xi_{0} p u_{1},
$$


therefore, $p u_{1}=0$. By induction, $u_{1} \in \operatorname{Span}(v)$, where $v$ is the generator of $\operatorname{Ind}_{\mathfrak{p}}^{\mathfrak{g}}(\lambda)$. Since $\xi_{0} \perp \mathfrak{p}_{\overline{1}}$, it follows that $\left[p, \xi_{0}\right] u_{1}=f_{\lambda}\left(p, \xi_{0}\right) u_{1}=0$. Therefore, $p u_{0}=0$ and $u_{0} \in \operatorname{Span}(v)$. Hence, $u \in \operatorname{Span}\left(v, \xi_{0} v\right)$.

ii) Let us show now that the weight of $\xi_{0} v$ with respect to $\mathfrak{g}_{0}$ is also equal to $\lambda$. If $\overline{\xi_{0}}=0$, all is clear. So let $\xi_{0} \neq 0$. Since $\left[x, \xi_{0}\right] \perp \mathfrak{p}_{\overline{1}}$ for any $x \in \mathfrak{g}_{\overline{0}}$, it follows that $\left[x, \xi_{0}\right]=\mu(x) \xi_{0}+p$ for some $p \in \mathfrak{p}_{\overline{1}}$. Furthermore, $\left[x,\left[\xi_{0}, \xi_{0}\right]=2\left[\left[x, \xi_{0}\right], \xi_{0}\right]\right.$; hence,

$$
\begin{aligned}
0 & =\lambda\left(\left[x,\left[\xi_{0}, \xi_{0}\right]\right]\right)=2 \lambda\left(\left[\mu(x) \xi_{0}+p, \xi_{0}\right]\right) \\
& =2 \mu(x) \lambda\left(\left[\xi_{0}, \xi_{0}\right]\right)+2 \lambda\left(\left[p, \xi_{0}\right]\right)=2 \mu(x) \lambda\left(\left[\xi_{0}, \xi_{0}\right]\right) .
\end{aligned}
$$

But $\lambda\left(\left[\xi_{0}, \xi_{0}\right]\right) \neq 0$, so, $\mu(x)=0$ and the weight of $\xi_{0} v$ is equal to $\lambda$.

iii) $\mathfrak{h}_{\overline{1}}^{\perp} \subset \mathfrak{h}_{\overline{1}}$. Then the restriction of the form $f_{\lambda}$ onto $\mathfrak{h}_{\overline{1}}$ is of rank two less than that of $f_{\lambda}$ itself.

Select $\xi \notin \mathfrak{h}_{\overline{1}}$ and set $F_{1}=F \cap \operatorname{Span}(\xi)^{\perp}$. Let $\mathfrak{n}$ be the kernel of $f_{\lambda}$. Then

$$
\operatorname{dim} \mathfrak{h}_{\overline{1}}+\operatorname{dim} \mathfrak{h}_{\overline{1}}^{\perp}=\operatorname{dim} \mathfrak{g}_{\overline{1}}+\operatorname{dim} \mathfrak{n},
$$

so $\operatorname{dim} \mathfrak{h}_{\overline{1}}^{\perp}=\operatorname{dim} \mathfrak{n}+1$. Therefore, there exists an element $\eta \in \mathfrak{h}_{\overline{1}}^{\perp} \cap F$ and such that $\eta \notin \mathfrak{n}$. Clearly, $f_{\lambda}(\xi, \eta) \neq 0$ and $\mathfrak{p}_{\overline{1}}^{\perp} \subset \mathfrak{h}_{\overline{1}}$. Therefore,

$$
F=F_{1} \oplus \operatorname{Span}(\eta), \quad \xi \perp F_{1} \text { and } f_{\lambda}(\xi, \eta) \neq 0 .
$$

Let

$$
u=u_{0}+\xi u_{1}, \text { where } u \in \operatorname{Ind}_{\mathfrak{p}}^{\mathfrak{g}}(\lambda), u_{0}, u_{1} \in \operatorname{Ind}_{\mathfrak{p}}^{\mathfrak{h}}(\lambda) \text { and } p u=0 \text { for any } p \in F .
$$

Then

$$
0=p u=p u_{0}+[p, \xi] u_{1}+\xi p u_{1},
$$

hence, $p u_{1}=0$ and by induction $u_{1} \in \operatorname{Span}\left(v, \xi_{0} v\right)$. Thanks to ii) $[p, \xi] u_{1}=$ $f_{\lambda}(p, \xi) u_{1}$ and if $p \in F_{1}$, then $f_{\lambda}(p, \xi) u_{1}=0$; hence, $p u_{0}=0$ for any $p \in F_{1}$. By induction we deduce that $u_{0} \in \operatorname{Span}\left(v, \xi_{0} v\right)$. Further on,

$$
0=\eta u=\eta u_{0}+\eta \xi u_{1}=[\eta, \xi] u_{1}=f_{\lambda}(\eta, \xi) u_{1}
$$

and since $f_{\lambda}(\eta, \xi) \neq 0$, then $u_{1}=0$ and $u=u_{0} \in \operatorname{Span}\left(v, \xi_{0} v\right)$.

3.2. Corollary. If $\mathfrak{h}=\mathfrak{g}_{\overline{0}} \oplus \mathfrak{h}_{\overline{1}}$ is a polarization for $\lambda$, then $\operatorname{Ind}_{\mathfrak{h}}^{\mathfrak{g}}(\lambda)$ is an irreducible module.

Proof. Observe that irreducibility is equivalent to the absence of vectors annihilated by $\mathfrak{b}_{\overline{1}}$ that do not lie in $\operatorname{Span}\left(v, \xi_{0} v\right)$.

3.3. Corollary. Heading 1) of Lemma 1.2 and heading 4) of the Main Theorem 1.3 hold.

3.4. Corollary. Let $U$ be an irreducible finite dimensional $\mathfrak{g}$-module. Then $U=$ $\operatorname{Ind}_{\mathfrak{h}}^{\mathfrak{g}}(\lambda)$ for some $\lambda \in L$ and a polarization $\mathfrak{h}$.

Proof. Induction on $\operatorname{dim} \mathfrak{g}_{\overline{1}}$. If $\mathfrak{g}=\mathfrak{g}_{\overline{0}}$, then this is Lie's theorem. Let $\mathfrak{k} \subset \mathfrak{g}$ and $\operatorname{dim} \mathfrak{g}_{\overline{1}} / \mathfrak{k}_{\overline{1}}=1$. 
Let $U$ be irreducible as a $\mathfrak{k}$-module. Then there exist $\lambda \in L$ and a polarization $\mathfrak{h} \subset \mathfrak{k}$ for $\lambda \in L$ such that $U=\operatorname{Ind}_{\mathfrak{h}}^{\mathfrak{k}}(\lambda)$. If $\mathfrak{h}$ were a polarization for $\lambda$ in $\mathfrak{g}$, too, then by Corollary 3.2 the representation

$$
\operatorname{Ind}_{\mathfrak{h}}^{\mathfrak{g}}(\lambda)=\operatorname{Ind}_{\mathfrak{k}}^{\mathfrak{g}}\left(\operatorname{Ind}_{\mathfrak{h}}^{\mathfrak{k}}(\lambda)\right)
$$

would have been irreducible contradicting Lemma 2.6.

Let $\hat{\mathfrak{h}} \supset \mathfrak{h}$ be a polarization for $\lambda$ in $\mathfrak{g}$ and $\xi \in \hat{\mathfrak{h}}$ so that $\xi \notin \mathfrak{h}$. If $v$ is an element of $\operatorname{Ind}_{\mathfrak{h}}^{\mathfrak{k}}(\lambda)$ as the one described in 3.1 and $p \in \mathfrak{h}_{\overline{1}}$, then

$$
\begin{gathered}
p \xi v=[p, \xi] v=f_{\lambda}(p, \xi) v=0, \\
\xi \xi v=\frac{1}{2}[\xi, \xi] v=\frac{1}{2} f_{\lambda}(\xi, \xi) v=0 .
\end{gathered}
$$

Therefore, there exists a non-zero $\mathfrak{g}$-module homomorphism $\operatorname{Ind}_{\hat{\mathfrak{h}}}^{\mathfrak{g}} \lambda \longrightarrow \operatorname{Ind}_{\mathfrak{h}}^{\hat{\mathfrak{k}}}(\lambda)=U$ and since both modules are irreducible, this is an "odd isomorphism," i.e., the composition of an isomorpism with the change of parity.

Now let $U$ be reducible as a $\mathfrak{k}$-module. Then by Lemma $2.7 U=\operatorname{Ind}_{\mathfrak{k}}^{\mathfrak{g}} V$, and, by induction, $V=\operatorname{Ind}_{\mathfrak{h}}^{\mathfrak{k}}(\lambda)$ for a polarization $\mathfrak{h} \subset \mathfrak{k}$ and $\lambda \in L$. If $\mathfrak{h}$ is not a polarization for $\lambda$ in $\mathfrak{g}$, then let $\hat{\mathfrak{h}} \supset \mathfrak{h}$ be a polarization. We have a non-zero $\mathfrak{g}$-module homomorphism $U=\operatorname{Ind}_{\mathfrak{h}}^{\mathfrak{g}}(\lambda) \longrightarrow \operatorname{Ind}_{\hat{\mathfrak{h}}}^{\mathfrak{g}}(\lambda)$ and since both modules are irreducible, this is an isomorphism which is impossible because $\operatorname{dim} \operatorname{Ind}_{\hat{\mathfrak{h}}}^{\mathfrak{g}}(\lambda)<\operatorname{dim} \operatorname{Ind}_{\mathfrak{h}}^{\mathfrak{g}}(\lambda)$. Therefore, $\mathfrak{h}$ is a polarization for $\lambda$ in $\mathfrak{g}$ and $U=\operatorname{Ind}_{\mathfrak{h}}^{\mathfrak{g}}(\lambda)$.

3.5. Corollary. Heading 1) of Theorem holds.

3.6. A subsuperalgebra subordinate for $\lambda \in L$. Recall (see $[\mathrm{K}]$ p. 79) that if

$$
\mathfrak{g}_{\lambda}=\left\{g \in \mathfrak{g} \mid \lambda\left(\left[g, g_{1}\right]\right)=0 \text { for all } g_{1} \in \mathfrak{g}\right\},
$$

then a subalgebra $\mathfrak{p} \subset \mathfrak{g}$ is called subordinate to $\lambda$ if $\lambda([\mathfrak{p}, \mathfrak{p}])=0$ and $\mathfrak{p} \supset \mathfrak{g}_{\lambda}$.

Corollary. Let $\lambda \in L, \mathfrak{p}$ a subalgebra subordinate to $\lambda$. Then $\operatorname{Ind}_{\mathfrak{p}}^{\mathfrak{g}}(\lambda)$ is irreducible if and only if $\mathfrak{p}$ is a polarization for $\lambda$.

\section{Classification of MOdules $\operatorname{Ind}_{\mathfrak{h}}^{\mathfrak{g}}(\lambda)$}

4.1. Lemma. If $(\lambda, \mathfrak{h}) \sim(\mu, \mathfrak{t})$, then $\mathfrak{h}$ is a polarization for $\mu$.

Proof. By $2.5 \mathfrak{h}$ is a polarization for $\lambda-\theta_{\mathfrak{h}}$. Since $\lambda-\theta_{\mathfrak{h}}=\mu-\theta_{\mathfrak{t}}$, then $\mathfrak{t}$ is also a polarization for $\lambda-\theta_{\mathfrak{h}}$. Let $\mathfrak{n}$ be the kernel of $f_{\lambda-\theta_{\mathfrak{h}}}$, then $\mathfrak{t} \supset \mathfrak{n}$. Hence, $\mathfrak{g}_{\mathfrak{1}} / \mathfrak{t}$ is a subfactor of $\mathfrak{g}_{1} / \mathfrak{n}$. Therefore, by Lemma 2.5 we see that $\mathfrak{h}$ is a polarization for $\mu=\left(\lambda-\theta_{\mathfrak{h}}\right)+\theta_{\mathfrak{t}}$.

4.2. Proof of heading 3) of Theorem. Let $(\lambda, \mathfrak{h}) \sim(\mu, \mathfrak{t})$. We will carry the proof out by induction on $k=\operatorname{dim} \mathfrak{h} /(\mathfrak{h} \cap \mathfrak{t})$. If $k=0$ the statement is obvious. Let $k=1$, then, obviously, $\operatorname{dim} \mathfrak{t} /(\mathfrak{h} \cap \mathfrak{t})=1$. Consider the space $\mathfrak{h}+\mathfrak{t}$. By Lemma $4.1 \mathfrak{t}$ is a polarization for $\lambda$ and, therefore, the kernel of $f_{\lambda}$ on the subspace $\mathfrak{h}+\mathfrak{t}$ is equal to $\mathfrak{h} \cap \mathfrak{t}$.

Let $\xi \in \mathfrak{h}$ and $\eta \in \mathfrak{t}$ be such that $\bar{\xi} \in \mathfrak{h} /(\mathfrak{h} \cap \mathfrak{t}), \bar{\xi} \neq 0$ and $\bar{\eta} \in \mathfrak{t} /(\mathfrak{h} \cap \mathfrak{t}), \bar{\eta} \neq 0$. We may assume that $f_{\lambda}(\xi, \eta)=1$.

Let $v \in \operatorname{Ind}_{\mathfrak{h}}^{\mathfrak{g}}(\lambda)$ be as in Proposition 3.1. Then for $r \in \mathfrak{h} \cap \mathfrak{t}$ we have

$$
r \eta v=[r, \eta] v=\lambda([r, \eta]) v=0, \quad \eta \eta v=\frac{1}{2}[\eta, \eta] v=\frac{1}{2} \lambda([\eta, \eta]) v=0,
$$


i.e., $\mathfrak{t}_{\overline{1}}(\eta v)=0$ and, therefore, there exists a non-zero homomorphism $\operatorname{Ind}_{\mathfrak{t}}^{\mathfrak{g}}(\tilde{\mu}) \longrightarrow$ $\operatorname{Ind}_{\mathfrak{h}}^{\mathfrak{g}}(\lambda)$, where $\tilde{\mu}$ is the weight of $\eta v$.

Since $\operatorname{Ind}_{\mathfrak{h}}^{\mathfrak{g}}(\lambda)$ is irreducible and $\operatorname{dim} \operatorname{Ind}_{\mathfrak{t}}^{\mathfrak{g}}(\mu)=\operatorname{dim}_{\operatorname{Ind}_{\mathfrak{h}}}^{\mathfrak{g}}(\lambda)$, this homomorphism is an isomorphism. Let $g \in \mathfrak{g}_{\overline{0}}$. Then

$$
g(\eta v)=\eta(g v)+[g, \eta] v=\left[\lambda+\operatorname{tr}_{\mathfrak{t} /(\operatorname{t} \cap \mathfrak{h})} \text { ad } g\right] \eta v,
$$

i.e., $\tilde{\mu}=\lambda+\operatorname{tr}_{\mathfrak{t} /(\mathfrak{t} \cap \mathfrak{h})}$ ad $g$.

Since $\lambda \in L$, it follows that

$$
\begin{aligned}
& 0=\lambda([g,[\xi, \eta]])=\lambda([[g, \xi], \eta])+\lambda([\xi,[g, \eta]]) \\
& =\left(\operatorname{tr}_{\mathfrak{t} / \mathfrak{t} \cap \mathfrak{h}} \operatorname{ad} g+\operatorname{tr}_{\mathfrak{h} / \mathfrak{t} \cap \mathfrak{h}} \text { ad } g\right) \lambda([\xi, \eta]) \text {. }
\end{aligned}
$$

Since $\lambda\left([\xi, \eta]=1\right.$, it follows that $\operatorname{tr}_{\mathfrak{t} / \mathfrak{t} \cap \mathfrak{h}}$ ad $g=-\operatorname{tr}_{\mathfrak{h} / \mathfrak{t} \cap \mathfrak{h}}$ ad $g$, and

$$
\mu=\lambda-\theta_{\mathfrak{p}}-\theta_{\mathfrak{t}}=\lambda+\operatorname{tr}_{\mathfrak{t} / \mathfrak{t} \cap \mathfrak{h}}=\tilde{\mu},
$$

i.e., $\operatorname{Ind}_{\mathfrak{t}}^{\mathfrak{g}}(\mu) \cong \pi\left(\operatorname{Ind}_{\mathfrak{h}}^{\mathfrak{g}}(\lambda)\right)$.

Let $k>1$. On $\mathfrak{g}_{\overline{1}}$, consider the form $f_{\lambda}$. Let $\mathfrak{h}=\mathfrak{g}_{\overline{0}}+\mathfrak{h}_{\overline{1}}$ and $\mathfrak{t}=\mathfrak{g}_{\overline{0}} \oplus \mathfrak{t}_{\overline{1}}$. Select $F$ so that $\mathfrak{h}_{\overline{1}} \cap \mathfrak{t}_{\overline{1}} \subset F \subset \mathfrak{h}_{\overline{1}}, F \neq \mathfrak{h}_{\overline{1}}$ and $F \neq \mathfrak{h}_{\overline{1}} \cap \mathfrak{t}_{\overline{1}}$, where $F$ is a $\mathfrak{g}_{\overline{0}}$-submodule in $\mathfrak{g}_{\overline{1}}$. Set $\mathfrak{r}_{\overline{1}}=F+\left(F^{\perp} \cap \mathfrak{t}_{\overline{1}}\right)$. It is not difficult to verify that $\mathfrak{r}=\mathfrak{g}_{\overline{0}} \oplus \mathfrak{r}_{\overline{1}}$ is a polarization for $\lambda$. Set

$$
\nu(x)=\lambda(x)-\operatorname{tr}_{\mathfrak{h}_{\overline{1}} /\left(\mathfrak{h}_{\overline{1}} \cap \mathfrak{r}_{\overline{1}}\right)}(\operatorname{ad} x) .
$$

Since $\mathfrak{h}_{\overline{1}} /\left(\mathfrak{h}_{\overline{1}} \cap \mathfrak{r}_{\overline{1}}\right)$ is a subfactor in $\mathfrak{g}_{\overline{1}} / \mathfrak{n}$, where $\mathfrak{n}$ is the kernel of $f_{\lambda}$, it follows from Lemma 2.5 that $\mathfrak{n}$ is a polarization for $\nu$.

Since $\mathfrak{h}_{\overline{1}} \cap \mathfrak{r}_{\overline{1}} \supset F \supset \mathfrak{h}_{\overline{1}} \cap \mathfrak{t}_{\overline{1}}$, then $\operatorname{dim} \mathfrak{r}_{\overline{1}} /\left(\mathfrak{h}_{\overline{1}} \cap \mathfrak{r}_{\overline{1}}\right)<\operatorname{dim} \mathfrak{h}_{\overline{1}} /\left(\mathfrak{h}_{\overline{1}} \cap \mathfrak{t}_{\overline{1}}\right)$.

Further, the diagram of inclusions

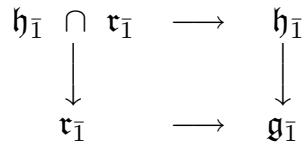

shows that

$$
2 \theta_{\mathfrak{h}}(x)-\operatorname{tr}_{\mathfrak{h}_{\overline{1}} /\left(\mathfrak{h}_{\overline{1}} \cap \mathfrak{r}_{\overline{1}}\right)} \text { ad }(x)=2 \theta_{\mathfrak{r}}(x)-\operatorname{tr}_{\mathfrak{r}_{\overline{1}} /\left(\mathfrak{r}_{\overline{1}} \cap \mathfrak{h}_{\overline{1}}\right)} \operatorname{ad}(x) .
$$

By duality, there exists a nondegenerate pairing

$$
\left(\mathfrak{h}_{\overline{1}} /\left(\mathfrak{h}_{\overline{1}} \cap \mathfrak{r}_{\overline{1}}\right)\right) \times\left(\mathfrak{r}_{\overline{1}} /\left(\mathfrak{h}_{\overline{1}} \cap \mathfrak{r}_{\overline{1}}\right)\right) \longrightarrow \mathbb{C}
$$

and since $\operatorname{tr}_{\mathfrak{h}_{\overline{1}} /\left(\mathfrak{h}_{\overline{1}} \cap \mathfrak{r}_{\overline{1}}\right)} \operatorname{ad}(x)=-\operatorname{tr}_{\mathfrak{r}_{\overline{1}} /\left(\mathfrak{h}_{\overline{1}} \cap \mathfrak{r}_{\overline{1}}\right)} \operatorname{ad}(x)$, then $\operatorname{tr}_{\mathfrak{r}_{\overline{1}} /\left(\mathfrak{h}_{\overline{1}} \cap \mathfrak{r}_{\overline{1}}\right)} \operatorname{ad}(x)=$ $-\theta_{\mathfrak{h}}(x)+\theta_{\mathfrak{r}}(x)$.

Thus,

$$
\nu(x)-\theta_{\mathfrak{r}}(x)=\lambda(x)+\operatorname{tr}_{\mathfrak{r}_{\overline{1}} /\left(\mathfrak{h}_{\overline{1}} \cap \mathfrak{r}_{\overline{1}}\right)} \operatorname{ad}(x)-\theta_{\mathfrak{r}}(x)=\lambda(x)-\theta_{\mathfrak{h}}(x),
$$

i.e., $(\lambda, \mathfrak{h}) \sim(\nu, \mathfrak{r})$ and, by induction, $\operatorname{Ind}_{\mathfrak{h}}^{\mathfrak{g}}(\lambda)=\operatorname{Ind}_{\mathfrak{r}}^{\mathfrak{g}}(\nu)$. Besides, $\nu-\theta_{\mathfrak{r}}=\lambda-\theta_{\mathfrak{h}}=$ $\mu-\theta_{\mathfrak{t}}$ and $\mathfrak{t}_{\overline{1}} \cap \mathfrak{r}_{\overline{1}} \supset F^{\perp} \cap \mathfrak{t}_{\overline{1}} \supset \mathfrak{t}_{\overline{1}} \cap \mathfrak{h}_{\overline{1}}$, where the latter inclusion is a strict one because $F \neq \mathfrak{h}_{\overline{1}}$; therefore,

$$
\operatorname{dim} \mathfrak{t}_{\overline{1}} / \mathfrak{t}_{\overline{1}} \cap \mathfrak{r}_{\overline{1}}<\operatorname{dim} \mathfrak{t}_{\overline{1}} /\left(\mathfrak{t}_{\overline{1}} \cap \mathfrak{h}_{\overline{1}}\right) .
$$

By induction, $\operatorname{Ind}_{\mathfrak{r}}^{\mathfrak{g}}(\nu) \cong \operatorname{Ind}_{\mathfrak{t}}^{\mathfrak{g}}(\mu)$, therefore, $\operatorname{Ind}_{\mathfrak{h}}^{\mathfrak{g}}(\lambda)=\operatorname{Ind}_{\mathfrak{t}}^{\mathfrak{g}}(\mu)$.

Conversely, let $\operatorname{Ind}_{\mathfrak{h}}^{\mathfrak{g}}(\lambda) \cong \operatorname{Ind}_{\mathfrak{t}}^{\mathfrak{g}}(\mu)$. Then $\lambda=\mu+\lambda_{1}+\cdots+\lambda_{k}$, where the $\lambda_{i}$ are the weights of $\mathfrak{g}_{\overline{1}} / \mathfrak{t}_{\overline{1}}$. Therefore, by Lemma $2.5 \mathfrak{t}$ is a polarization for $\lambda$ and, thanks to section 4.1 , for $\tilde{\mu}=\lambda-\theta_{\mathfrak{h}}+\theta_{\mathfrak{t}}$, too. Since $\tilde{\mu}-\theta_{\mathfrak{t}}=\lambda-\theta_{\mathfrak{h}}$, then by the above $\operatorname{Ind}_{\mathfrak{t}}^{\mathfrak{g}}(\tilde{\mu})=\operatorname{Ind}_{\mathfrak{h}}^{\mathfrak{g}}(\lambda)=\operatorname{Ind}_{\mathfrak{t}}^{\mathfrak{g}}(\mu)$. Let $\tilde{v} \in \operatorname{Ind}_{\mathfrak{t}}^{\mathfrak{g}}(\tilde{\mu})$ be as in Proposition 3.1 
and $\mathfrak{t}_{\overline{1}} \tilde{v}=0$. By $4.1 \tilde{v} \in \operatorname{Span}\left(v, \xi_{0} v\right)$, where $v \in \operatorname{Ind}_{\mathfrak{t}}^{\mathfrak{g}}(\mu)$ be as in Proposition 3.1; therefore, $\tilde{\mu}=\mu$ and $(\mu, \mathfrak{t}) \sim(\lambda, \mathfrak{h})$.

4.3. Corollary. Heading 2) of Theorem and heading 2) of Lemma 1.2 hold.

Proof. Due to section 2.5 it is clear that $\mathfrak{h}$ is a polarization for $\lambda+\theta_{\mathfrak{h}}$ and, therefore, $I_{\mathfrak{h}}^{\mathfrak{g}}(\lambda)$ is irreducible. If $\mathfrak{t}$ is another polarization for $\lambda$, then by section 4.2

$$
I_{\mathfrak{t}}^{\mathfrak{g}}(\lambda)=\operatorname{Ind}_{\mathfrak{t}}^{\mathfrak{g}}\left(\lambda+\theta_{\mathfrak{t}}\right)=\operatorname{Ind}_{\mathfrak{h}}^{\mathfrak{g}}\left(\lambda+\theta_{\mathfrak{h}}\right)=I_{\mathfrak{h}}^{\mathfrak{g}}(\lambda) .
$$

If $U$ is irreducible, then by section $3.4 U \cong I_{\mathfrak{h}}^{\mathfrak{g}}(\lambda)$ for some $\lambda$ and $\mathfrak{h}$.

If $I(\lambda)=I(\mu)$, then $\operatorname{Ind}_{\mathfrak{h}}^{\mathfrak{g}}\left(\lambda+\theta_{\mathfrak{h}}\right) \cong \operatorname{Ind}_{\mathfrak{t}}^{\mathfrak{g}}\left(\mu+\theta_{\mathfrak{t}}\right)$ and by section 4.2

$$
\lambda=\lambda+\theta_{\mathfrak{h}}-\theta_{\mathfrak{h}}=\mu+\theta_{\mathfrak{t}}-\theta_{\mathfrak{t}}=\mu \text {. }
$$

Proof of heading 3) of Lemma 1.2. Let us prove now that $I_{\mathfrak{p}}^{\mathfrak{g}}(\lambda) \cong C I_{\mathfrak{p}}^{\mathfrak{g}}(\lambda)$. To this end, make use of the isomorphisms $\left(I_{\mathfrak{p}}^{\mathfrak{g}}(\lambda)\right)^{*} \cong C I_{\mathfrak{p}}^{\mathfrak{g}}(-\lambda)$ and $\left(I_{\mathfrak{p}}^{\mathfrak{g}}(\lambda)\right)^{*} \cong$ $I_{\mathfrak{p}}^{\mathfrak{g}}\left(-\lambda+2 \theta_{\mathfrak{p}}\right)$. The first of these isomorphisms follows from the definitions of the induced and coinduced modules.

Let us prove the other one. Select a basis $\xi_{1}, \ldots, \xi_{n}$ in the complement to $\mathfrak{p}_{\overline{1}}$ in $\mathfrak{g}_{\overline{1}}$ and consider the following filtration of $I_{\mathfrak{p}}^{\mathfrak{g}}(\lambda)$ :

$$
I_{0}=\operatorname{Span}(v) \subset I_{1}=\operatorname{Span}\left(v, \xi_{1} v, \xi_{2} v, \ldots, \xi_{n} v\right) \subset \cdots \subset I_{n}=I_{\mathfrak{p}}^{\mathfrak{g}}(\lambda),
$$

where $v$ is as in Proposition 3.1. It is clear that the elements $\xi_{1}, \xi_{2}, \ldots, \xi_{n}$ can be chosen so that each $I_{k}$ is a $\mathfrak{g}_{0}$-module. Let $l \in\left(I_{\mathfrak{p}}^{\mathfrak{g}}(\lambda)\right)^{*}$ be such that $l\left(I_{n}\right) \neq 0$ while $l\left(I_{n-1}\right)=0$. Then it is easy to verify that $\mathfrak{p}_{\overline{1}} l=0$ and the weight $l$ with respect to $\mathfrak{g}_{\overline{0}}$ is equal to $-\lambda+2 \theta_{\mathfrak{p}}$. Therefore, there exists a nonzero homomorphism $\varphi: I_{\mathfrak{p}}^{\mathfrak{g}}\left(-\lambda+2 \theta_{\mathfrak{p}}\right) \longrightarrow\left(I_{\mathfrak{p}}^{\mathfrak{g}}(\lambda)\right)^{*}$.

Since the dimensions of these modules are equal and the first of them is irreducible, $\varphi$ is an isomorphism. Hence,

$$
C I(\lambda)=C I_{\mathfrak{p}}^{\mathfrak{g}}\left(\lambda-\theta_{\mathfrak{p}}\right) \cong\left(I_{\mathfrak{p}}^{\mathfrak{g}}\left(-\lambda+\theta_{\mathfrak{p}}\right)\right)^{*}=I_{\mathfrak{p}}^{\mathfrak{g}}\left(\lambda-\theta_{\mathfrak{p}}+2 \theta_{\mathfrak{p}}\right)=I(\lambda) .
$$

\section{An EXAMPLE}

Let $\Lambda(2)=\mathbb{C}\left[\xi_{1}, \xi_{2}\right]$ be the Grassmann superalgebra on two indeterminates with the natural $\mathbb{Z} / 2$-grading (parity). In $\mathfrak{g l}(\Lambda(2))$, consider the linear hull $\mathfrak{g}$ of the operators

$$
\begin{gathered}
x=\xi_{1} \frac{\partial}{\partial \xi_{1}}, \quad y=\xi_{2} \frac{\partial}{\partial \xi_{1}}, \quad z=\xi_{1} \xi_{2}, \quad u=1 \\
\eta_{1}=\frac{\partial}{\partial \xi_{1}}, \quad \eta_{2}=\frac{\partial}{\partial \xi_{2}}-\xi_{1} \xi_{2} \frac{\partial}{\partial \xi_{1}} \\
\eta_{-1}=\xi_{1}, \quad \eta_{-2}=\xi_{2}
\end{gathered}
$$

where $f \in \Lambda(2)$ is identified with the operator of left multiplication by $f$. It is not difficult to verify that $\mathfrak{g}=\mathfrak{g}_{\overline{0}} \oplus \mathfrak{g}_{\overline{1}}$ is a Lie superalgebra. It is solvable since $\mathfrak{g}_{\overline{0}}$ is also. Moreover, $\left[\mathfrak{g}_{\overline{0}}, \mathfrak{g}_{\overline{0}}\right]=\operatorname{Span}(y, z)$ and $\left[\mathfrak{g}_{\overline{1}}, \mathfrak{g}_{\overline{1}}\right]=\mathfrak{g}_{\overline{0}}$.

Let $u^{*}, y^{*}, z^{*}, x^{*}$ be the basis of $\mathfrak{g}_{0}^{*}$ left dual $u, y, z, x$, respectively, and let $\lambda=u^{*}$. Then $\mathfrak{h}=\mathfrak{g}_{\overline{0}} \oplus \operatorname{Span}\left(\eta_{-1}, \eta_{-2}\right)$ and $\mathfrak{t}=\mathfrak{g}_{\overline{0}} \oplus \operatorname{Span}\left(\eta_{1}, \eta_{2}\right)$ are polarizations for $\lambda$. 
As is easy to verify, the characters of the irreducible factors of the $\mathfrak{g}_{\overline{0}}$-module $I_{\mathfrak{h}}^{\mathfrak{g}}(\lambda)$ are $\lambda$ and $\lambda-x^{*}$ whereas the characters of the irreducible factors of the $\mathfrak{g}_{0}$-module $I_{\mathfrak{t}}^{\mathfrak{g}}(\lambda)$ are $\lambda$ and $\lambda+x^{*}$. Hence, $I_{\mathfrak{h}}^{\mathfrak{g}}(\lambda) \not I_{\mathfrak{t}}^{\mathfrak{g}}(\lambda)$.

Moreover, $\lambda-\left(\lambda-x^{*}\right)=x^{*}$ but $x^{*}\left(\left[\mathfrak{g}_{\overline{1}}, \mathfrak{g}_{\overline{1}}\right]\right) \neq 0$ contradicting the statement of Theorem 7 of $[\mathrm{K}]$.

The error in the proof of Theorem 7 of $[\mathrm{K}]$ is not easy to find: it is an incorrect induction in the proof of heading a) on p. 80. Namely, if, in notations of $[\mathrm{K}]$, the subalgebra $H$ is of codimension $(0,1)$, then the irreducible factors of $W$ considered as $G_{0}$-modules belong by the inductive hypothesis to one class from $L / L_{0}^{H}$, where

$$
L_{0}^{H}=\left\{\lambda \in \mathfrak{g}^{*}: \lambda([H, H])=0\right\},
$$

NOT to one class from $L / L_{0}^{G}$ as stated on p. 80 , line 13 of $[\mathrm{K}]$.

\section{REFERENCES}

[BL] Bernstein J. and Leites D., The superalgebra $Q(n)$, the odd trace and the odd determinant, C. R. Acad. Bulgare Sci. 35 (1982), no. 3, 285-286. MR 84c:17003

[D] Dixmier J., Enveloping algebras, Revised reprint of the 1977 translation, Graduate Studies in Mathematics, 11, American Mathematical Society, Providence, RI, 1996. MR 97c:17010

[K] Kac V., Lie superalgebras, Adv. Math. 26 (1977), 8-96. MR 58:5803

[L] Leites D. (ed.), Seminar on supermanifolds \#22, Reports of the Department of Mathematics of Stockholm University, 1988-4, 1-12.

[Shch] Shchepochkina I. M., Maximal solvable subalgebras of the Lie superalgebras $\mathfrak{g l}(m \mid n)$ and $\mathfrak{s l}(m \mid n)$. (Russian) Funktsional. Anal. i Prilozhen. 28 (1994), no. 2, 92-94; translation in Functional Anal. Appl. 28 (1994), no. 2, 147-149. MR 95b:17013

On leave of absence from Balakovo Institute of Technique of Technology and Control, Branch of Saratov State Technical University, Russia

Department of Mathematics, University of Stockholm, Roslagsv. 101, Kräftriket hus 6, S-106 91, Stockholm, Sweden

E-mail address: mleites@matematik.su.se (subject: for Sergeev) 